\title{
Habilidades Sociais e Problemas de Comportamento de Crianças com Altas Habilidades/Superdotação
}

\author{
Ana Paula de Oliveira ${ }^{1}$ \\ ${ }^{1}$ Universidade Estadual Paulista, SP, Brasil. \\ Vera Lucia Messias Fialho Capellini ${ }^{1}$ \\ ${ }^{1}$ Universidade Estadual Paulista, SP, Brasil.
}

\author{
Olga Maria Piazentin Rolim Rodrigues ${ }^{1}$ \\ ${ }^{1}$ Universidade Estadual Paulista, SP, Brasil. \\ Alessandra Turini Bolsoni-Silva ${ }^{1}$ \\ ${ }^{1}$ Universidade Estadual Paulista, SP, Brasil.
}

\begin{abstract}
Resumo: O estudo das habilidades sociais contribui para a compreensão das relações interpessoais, embora ainda haja poucos estudos que relacionem esse tema a crianças com altas habilidades/superdotação (AH/SD). Portanto, os objetivos deste estudo foram: a) descrever e comparar as habilidades sociais de crianças com AH/SD sob o ponto de vista da criança e de seus respectivos pais/responsáveis e professoras; b) descrever e comparar as habilidades sociais e os problemas de comportamento de crianças com AH/SD sob o ponto de vista de seus pais/responsáveis e professoras, considerando o sexo e comparando a importância das habilidades sociais para os dois grupos; e c) descrever a competência acadêmica das crianças segundo as professoras. Participaram dez pais/responsáveis, oito professoras e dez crianças estudantes do ensino fundamental identificados com AH/SD. Os participantes responderam ao questionário Sistema de Avaliação de Habilidades Sociais (SSRS) versão para pais, professores e alunos, e ao Questionário de Respostas Socialmente Habilidosas (QRSH), versão para pais e professores. Os resultados apontaram que as crianças apresentaram habilidades sociais acima da média para seus pais e professores, e abaixo da média no autorrelato das crianças. Entretanto, há indicativos de necessidade de atenção preventiva e de intervenção nos fatores que apresentaram déficits sociais de acordo com o relato dos três grupos de participantes em: autocontrole/civilidade; cooperação/empatia; assertividade/desenvoltura social; problemas de comportamento externalizantes e internalizantes; e hiperatividade. Tais dados possibilitam que sejam programadas intervenções preventivas, conforme indicação dos pais/responsáveis e professoras, condizentes com a necessidade apontada pelas próprias crianças para a promoção de habilidades sociais.
\end{abstract}

Palavras-chave: Altas Habilidades, Superdotação, Habilidades Sociais, Problemas de Comportamento, Múltiplos Informantes.

\section{Social Skills and Behavioral Problems of High-Ability/Gifted Children}


and teachers. For parents and teachers, children's social skills were considered above average, whereas for themselves they were considered below average. However, the three groups indicated social deficits in some factors that require preventive attention and interventions, namely: self-control/civility; cooperation/empathy; social assertiveness/resourcefulness; external and internalizing behavior problems; and hyperactivity. These findings enable the development of preventive interventions aimed at promoting social skills among HA/G children based on the reports of parents/guardians, teachers and children themselves.

Keywords: High Abilities, Giftedness, Social Skills, Behavioral Problems, Multiple Informants.

\title{
Habilidades Sociales y Problemas Conductuales de Niños con Altas Habilidades/Superdotados
}

\begin{abstract}
Resumen: Los estudios de las habilidades sociales contribuyen a la comprensión de las relaciones interpersonales, pero todavía son pocos los que relacionan este tema al de niños con altas habilidades/superdotados (AH/SD). Teniendo en cuenta esto, los objetivos de este estudio fueron: a) describir y comparar las habilidades sociales de niños con AH/SD desde el punto de vista del niño y de sus respectivos padres/responsables y profesoras; b) describir y comparar las habilidades sociales y los problemas conductuales de niños con AH/SD desde el punto de vista de sus padres/responsables y profesoras, considerando el sexo y la importancia de las habilidades sociales para ambos grupos; y c) describir la competencia académica de los niños según las profesoras. Participaron 10 padres/responsables, siendo ocho profesoras y diez alumnos de educación primaria de una escuela pública identificados con AH/SD. Los participantes respondieron al cuestionario Sistema de Evaluación de las Habilidades Sociales (SSRS), versión para padres, profesores y alumnos, y al Cuestionario de Respuestas Socialmente Habilidosas (CRSH), versión para profesores. Los resultados indicaron que los niños presentaban habilidades sociales mayor del promedio para sus padres y maestros y por debajo del promedio en el autoinforme de los niños. Sin embargo, se observó la necesidad de atención preventiva e intervención en los factores que presentaron déficits sociales de acuerdo con el relato de los tres grupos de participantes en: autocontrol/cortesía; cooperación/empatía; asertividad social/ingenio; problemas de conducta externos e internalizantes e hiperactividad. Estos datos permiten programar intervenciones preventivas, conforme indicación de los padres/responsables y profesoras y la necesidad de los propios niños, para la promoción de habilidades sociales.
\end{abstract}

Palabras clave: Altas Habilidades, Superdotados, Habilidades Sociales, Problemas de Conducta, Informantes Múltiples.

\section{Introdução}

As habilidades sociais são consideradas um constructo descritivo dos comportamentos sociais disponíveis no repertório do indivíduo (Del Prette \& Del Prette, 2017). Em dado contexto situacional-cultural, apresentam alta probabilidade de produzir reforçadores para a pessoa e minimizar a estimulação aversiva, o que contribui para a qualidade e a efetividade do relacionamento com o outro. Um bom repertório de habilidades sociais é condição necessária, mas não suficiente, para a competência social. Segundo os autores, a competência social é entendida como um constructo avaliativo do desempenho efetivamente apresentado pelo indivíduo diante de tarefas sociais específicas (Del Prette \& Del Prette, 2017). Portanto, uma pessoa socialmente competente "expressa uma leitura adequada do ambiente social, ou seja, decodifica corretamente os desempenhos esperados, 
valorizados e efetivos para o indivíduo em sua relação com os demais" (Del Prette \& Del Prette, 2008, p. 33).

Os déficits de habilidades sociais são a ausência de comportamentos esperados em tarefas ou condições específicas, ou, ainda, comportamentos que não atingem determinados critérios de frequência ou de proficiência nessas tarefas (McFall, 1982). Essas possibilidades caracterizam diferentes tipos de déficit: de aquisição, de desempenho e de fluência. $\mathrm{O}$ déficit de aquisição ocorre quando o indivíduo tem desconhecimento sobre como desempenhar uma dada habilidade social em determinada tarefa. O déficit de desempenho é a falha durante a execução de uma habilidade social, ainda que a pessoa saiba como desempenhá-la (Gresham, 2009). Já o déficit de fluência acontece quando a habilidade é emitida sem a proficiência necessária para produzir os resultados esperados (Del Prette \& Del Prette, 2013).

Além das habilidades sociais promoverem o desenvolvimento, é observado que, quando presentes no repertório da criança, o desempenho acadêmico delas é melhor (Del Prette \& Del Prette, 2013). Os comportamentos habilidosos também previnem o surgimento de problemas de comportamento na medida em que possibilitam que as crianças e adolescentes interajam mais positivamente com colegas, familiares e professores, aumentando a chance de acesso a reforçamento social, como atenção e elogios (BolsoniSilva, Marturano, Pereira, \& Manfrinato, 2006). Os problemas de comportamento podem ser internalizantes e/ou externalizantes (Patterson, Reid, \& Dishion, 2002). Os internalizantes são evidenciados por retraimento, ansiedade, depressão, sentimentos de inferioridade e queixas somáticas, levando as crianças e adolescentes a se privarem de interagir com o ambiente. Crianças com esse tipo de comportamento podem evitar iniciar uma interação com pares ou adultos, ou mesmo responder a eles, dificultando ou até impedindo interações que poderiam resultar em novas aprendizagens. Em contrapartida, os externalizantes são caracterizados por agitação, hiperatividade, impulsividade e agressão, além de características antissociais e desafiantes. Tais comportamentos podem gerar conflitos em seu ambiente e provocar rejeição de pais, colegas e professores (Patterson, Reid, \& Dishion, 2002).

Del Prette e Del Prette $(2009,2017)$ afirmam que há instrumentos e procedimentos que avaliam as habilidades sociais, mas não necessariamente a competência social, assim como o contrário. Segundo os autores, os instrumentos e procedimentos de relato produzem indicadores de habilidades sociais. Por sua vez, os procedimentos de observação direta fornecem indicadores de competência social, ainda que produzam, da mesma forma, indicadores de habilidades sociais. Assim, os autores enfatizam que, para avaliar o repertório habilidoso, é preciso considerar em quais contextos (por exemplo, familiar, escolar) e com que tipo de interlocutores (por exemplo, familiares, professores) o indivíduo apresenta recursos e/ou déficits de habilidades sociais. A partir daí, pode-se comparar seu repertório com o de outros indivíduos da mesma cultura e perfil sociodemográfico, pois esses eventos constituem contingências que podem determinar repertórios diferenciados, mas não necessariamente deficitários. Portanto, é recomendável que a avaliação seja multimodal, baseada em diferentes instrumentos e procedimentos, com diferentes informantes e contemplando contextos diversificados de interação social (Del Prette \& Del Prette, 2009, 2013).

Tais avaliações permitem mapear de forma mais abrangente os déficits e as habilidades sociais e, assim, nortear intervenções quando necessárias (Barreto, Freitas, \& Del Prette, 2011). Por exemplo, mães e professores podem julgar de modo distinto os comportamentos dos estudantes, pois os ambientes em que estão inseridos são distintos e apresentam demandas e exigências próprias. Tal fato justifica avaliações em ambos os contextos para melhor compreender as interações estabelecidas entre os adultos e os estudantes, assim como os comportamentos sociais de cada um deles (Bandeira, Silva, Cordeiro, Del Prette, \& Del Prette, 2006; Bolsoni-Silva et al., 2006; Eklund, Tanner, Stoll, \& Anway, 2015).

Bolsoni-Silva et al. (2006) compararam avaliações de mães e professoras sobre habilidades sociais e problemas de comportamento de crianças pré-escolares, identificadas pelas professoras como apresentando problemas de comportamento ou comportamentos socialmente habilidosos. Os resultados apontaram que mães e professoras de crianças sem problemas de comportamento não diferiram na avaliação das habilidades sociais, mas diferiram quanto aos problemas, percebidos em nível mais alto pelas mães. Mães e professoras de crianças com problemas discordaram na avaliação dos problemas de comportamento e de habilidades sociais, pois as mães perceberam mais habilidades e menos problemas. Diferenças de gênero 
foram encontradas apenas para problemas de comportamento das crianças com problemas, que refere mais comportamentos externalizantes nos meninos e maior competência social nas meninas.

Bandeira et al. (2006) investigaram as características sociodemográficas da ocorrência de comportamentos problemáticos e suas relações com as habilidades sociais e dificuldades acadêmicas em uma amostra de 257 estudantes (matriculados entre a primeira e a quarta série do ensino fundamental), 185 pais e 12 professores utilizando a escala Social Skills Rating System (SSRS). Os resultados mostraram que a ocorrência de comportamentos problemáticos das crianças foi mais frequentemente observada pelos pais do que pelos professores. No que se refere ao sexo, os meninos apresentaram maior frequência de comportamentos problemáticos do que as meninas, tanto na avaliação dos pais quanto na das professoras. Os problemas de comportamento foram encontrados mais frequentemente em crianças de nível socioeconômico mais baixo e com desempenho acadêmico deficitário. A frequência de comportamentos problemáticos foi menor em crianças que apresentaram um nível mais adequado e elaborado de habilidades sociais.

Bolsoni-Silva e Loureiro (2016) compararam as habilidades sociais de crianças pré-escolares e escolares segundo pais e professores, considerando grupos diferenciados por problemas de comportamento e gênero. Os resultados indicaram que, para ambos, as crianças sem problemas são mais habilidosas, tanto na pré-escola como na idade escolar. Para os professores, em ambos os períodos as meninas foram mais habilidosas e, para pais e professores, os meninos apresentaram mais problemas de comportamento. Em geral, os professores identificaram mais habilidades sociais do que os pais. Percebeu-se, na pesquisa, que as habilidades sociais diferenciaram crianças com e sem problemas de comportamento, independentemente dos anos de estudo, enquanto o gênero teve forte poder discriminatório para os professores e menos influência sobre a avaliação dos pais. Nota-se que, nos estudos conduzidos com escolares e pré-escolares, houve diferenças entre as avaliações das mães e professores.

Em relação às altas habilidades/superdotação (AH/SD), pesquisas brasileiras na área têm se concentrado principalmente nas demandas da educação e das políticas públicas, a partir da identificação delas e de programas para estimular o desenvolvimento e a criatividade (Alencar, 2007; Chagas \& Fleith, 2010), com poucos estudos que abordem as características socioemocionais dessa população (França-Freitas, Del Prette, \& Del Prette, 2014, 2017; Loos-Sant'Ana \& Trancoso, 2014).

O conceito de pessoas com AH/SD proposto pela Política Nacional de Educação Especial na Perspectiva da Educação Inclusiva (Secretaria de Educação Especial, 2008) caracteriza-se pelo alto desempenho e/ou elevada potencialidade em qualquer dos seguintes aspectos, isolados ou combinados: capacidade intelectual geral, aptidão acadêmica específica, pensamento criativo e/ou produtivo, capacidade de liderança, talento especial para as artes e capacidade psicomotora. Renzulli $(2004,2018)$ descreve um conjunto de três aspectos na identificação da pessoa com AH/SD: habilidade acima da média, criatividade e envolvimento com a tarefa, representadas em três círculos interconectados que denominou de modelo dos três anéis. Renzulli (2014) descreve o termo habilidade acima da média tanto para habilidades gerais como para as específicas. Para o autor, a habilidade geral consiste na capacidade de processar informações, de integrar experiências que resultem em respostas apropriadas e adaptativas a novas situações e de se engajar em pensamento abstrato. A habilidade específica se refere à capacidade de adquirir conhecimento e técnica ou executar uma ou mais das atividades humanas interpretadas como de domínio superior.

Ainda segundo o autor, as crianças superdotadas e talentosas são aquelas que possuem ou são capazes de desenvolver esse conjunto de comportamentos e aplicá-los a qualquer área potencialmente valorizada do desempenho humano. Esse conjunto permite uma classificação de dois tipos de AH/SD: o acadêmico e o produtivo criativo.

Renzulli (1998) explica, ainda, que além dos aspectos do modelo dos três anéis, outros fatores devem ser considerados, pois podem explicar o que faz com que algumas pessoas apresentem comportamento de superdotação em certos momentos e/ou em determinadas circunstâncias. $\mathrm{O}$ autor destaca alguns fatores ambientais e familiares, dentre os quais, as características dos pais ou da família, como o nível socioeconômico, a escolaridade, as características de personalidade de um dos pais e um ambiente estimulador presente desde a infância. Segundo o autor, o ambiente educacional formal ao qual a criança 
foi exposta é determinante para a manifestação e o desenvolvimento da AH/SD.

Quantoàs características das pessoas com AH/SD, algumas pesquisas apontam que elas apresentam déficits nas áreas sociais e/ou emocionais (Alencar, 2007; Antipoff \& Campos, 2010; Chagas \& Fleith, 2010; Eren, Çete, Avcil, \& Baykara, 2018; Gokaydin \& Ozcan, 2018; Ogurlu, Yalin, \& Birben, 2018; Oliveira, Capellini, \& Rodrigues, 2020; Peterson, 2009).

Algumas características relacionadas às habilidades sociais e/ou emocionais encontradas pelos autores são: isolamento social, distanciamento do grupo de pares, sentir-se diferente, não se adaptar ao contexto social, insegurança, raiva por não atingir a perfeição, sentimentos de inadequação, ideação suicida, baixa autoestima, depressão, ansiedade, irritabilidade, hostilidade, comportamento agressivo e impulsividade.

Almeida, Rocha e Fonseca (2016) apontam que todas as crianças e jovens deparam-se com vários problemas ao longo de seu desenvolvimento. Porém, as com $\mathrm{AH} / \mathrm{SD}$, devido a suas características singulares, podem se deparar com várias questões emocionais e/ou sociais mais acentuadas ao longo de seu desenvolvimento. Gokaydin e Ozcan (2018) investigaram a opinião de dez psicólogos a respeito de problemas emocionais e sociais enfrentados por pessoas com AH/SD. Os resultados apontaram que as pessoas com AH/SD vivenciavam problemas sociais, como solidão, dificuldades nas relações com os pares e exclusões; e problemas emocionais, como perfeccionismo, transtornos de humor e ansiedade, solidão e introversão.

Entretanto, outras pesquisas contrariaram esses achados, pois revelam que essa população apresenta bom repertório de habilidades sociais e/ou emocionais (Bain, Choate, \& Bliss, 2006; Freitas \& Del Prette, 2013; França-Freitas, Del Prette, \&Del Prette, 2014, 2017; Galloway \& Porath, 1997; Loos-Sant'Ana \& Trancoso, 2014; Prieto, Ferrándiz, Ferrando, Sánchez, \& Bermejo, 2016; Richards, Encel, \& Shute, 2003; Versteynen, 2001). Entre as características, os autores indicaram, por exemplo, responsabilidade, assertividade, autocontrole, civilidade, expressão de sentimento positivo, bom humor, capacidade de resolução de problemas, competência social, habilidades de liderança e autoestima elevada.

Freitas e Del Prette (2013) avaliaram as habilidades sociais de estudantes segundo os seus professores por meio do Sistema de Avaliação de Habilidades Sociais (SSRS-BR) - versão professor. Participaram do estudo doze grupos de dez estudantes, com idade entre 6 e 14 anos, e seus professores, de escolas regulares e especiais, de diferentes categorias de necessidades educativas especiais: AH/SD, autismo, deficiência auditiva, deficiência intelectual leve, deficiência intelectual moderada, deficiência visual, desvio fonológico, dificuldades de aprendizagem, problemas de comportamento externalizantes, problemas de comportamento internalizantes, problemas de comportamento internalizantes e externalizantes e transtorno de déficit de atenção e hiperatividade (TDAH). Os estudantes com AH/SD apresentaram escores significativamente maiores na escala global e/ ou nas subescalas de habilidades sociais em relação a outros seis grupos do estudo. Nas subescalas "responsabilidade/cooperação", o desempenho do grupo com AH/SD foi melhor do que os grupos com TDAH, problemas de comportamento externalizantes e problemas de comportamento internalizante e externalizante. Na subescala "asserção positiva", foi melhor do que os grupos com autismo e problemas de comportamento internalizantes. Em "autocontrole", o desempenho do grupo com AH/SD foi melhor do que os grupos com TDAH, autismo, problemas de comportamento externalizantes e problemas de comportamento internalizantes e externalizantes. Em "autodefesa", o desempenho do grupo com AH/SD foi melhor do que os grupos com autismo e dificuldades de aprendizagem. Trata-se, portanto, do grupo que apresentou o melhor repertório de habilidades sociais em comparação com os demais. Esse conjunto de resultados permite concluir que os estudantes com AH/SD apresentaram reservas comportamentais em uma grande variedade de habilidades sociais, segundo seus professores.

França-Freitas, Del Prette e Del Prette (2014) avaliaram as habilidades sociais de estudantes com e sem AH/SD por meio do autorrelato no Sistema de Avaliação de Habilidades Sociais (SSRS-BR) - versão estudante. Participaram do estudo 394 crianças, com entre 8 e 12 anos, dentre as quais 269 identificadas com AH/SD. Os resultados indicaram um repertório mais elaborado de habilidades sociais para os estudantes com AH/SD nas seguintes áreas: "responsabilidade", "assertividade", "autocontrole", "civilidade" e "expressão de sentimento positivo", porém, menos elaborado em "empatia".

Loos-Sant'Ana e Trancoso (2014) investigaram a autoavaliação de 45 estudantes, de 8 a 12 anos, com AH/SD, a respeito de suas habilidades sociais e 
desempenho acadêmico. Apenas quatro dos 45 participantes relataram ter repertório social abaixo da média, poucos relataram ansiedade em relação a seu desempenho acadêmico, a maioria relatou desempenho na média ou ligeiramente acima da média e a minoria relatou desempenho acima da média. Neste estudo, os resultados indicaram que os estudantes apresentam autoavaliação positiva em relação ao repertório social, mais do que em relação ao desempenho acadêmico.

Galloway e Porath (1997) investigaram as habilidades sociais de 23 estudantes com AH/SD, entre seis e doze anos, a partir do relato de seus pais e professores, utilizando o Sistema de Avaliação de Habilidades Sociais (SSRS) - versão professor e pais. Os resultados apontaram que não houve diferenças significativas na comparação das avaliações de professores e pais na escala geral, mas sim nas subescalas de cooperação e assertividade. Os professores avaliaram a cooperação dos estudantes mais frequentemente do que os pais, enquanto os pais avaliaram a assertividade mais frequentemente do que os professores.

Gauck e Trommsdorff (2009) compararam problemas de comportamento de trinta crianças com $\mathrm{AH} / \mathrm{SD}$ e $24 \mathrm{sem} \mathrm{AH} / \mathrm{SD}$, segundo a perspectiva delas mesmas e de seus pais e professores. Os resultados evidenciaram que os problemas não diferiram entre os dois grupos de crianças.

Eklund, Tanner, Stoll e Anway (2015) avaliaram características de risco emocional e comportamental em 1.206 crianças entre 5 e 12 anos, com e sem AH/SD, a partir do relato de seus pais e professores. Os resultados demonstraram que, tanto para os pais quanto para os professores, as crianças sem $\mathrm{AH} / \mathrm{SD}$ apresentavam mais riscos. A comparação entre o relato de pais e professores de crianças com AH/SD não revelou diferença significativa de riscos. Entretanto, ao comparar o relato deles sobre crianças sem AH/SD, os professores avaliaram mais riscos do que os pais. Entre as crianças indicadas com riscos, os pais identificaram um maior número de crianças com AH/SD do que os professores e, também, com taxas altas de comportamentos internalizantes entre as com AH/SD. Os autores concluíram que, apesar de não haver diferença significativa entre os grupos, ambos explicitaram características de riscos emocionais e comportamentais.

França-Freitas, Del Prette e Del Prette (2017) avaliaram o poder preditivo entre habilidades sociais e bem-estar subjetivo de 269 crianças, de 8 a 12 anos, com AH/SD. Os resultados demonstraram que um repertório elaborado de habilidades sociais está associado à percepção de maior bem-estar subjetivo, que refere felicidade e satisfação, enfatizando a importância das habilidades sociais na qualidade de vida e, consequentemente, como fator de proteção.

Eren et al. (2018) compararam características emocionais e comportamentais de estudantes com idade entre 9 e 12 anos, 49 com AH/SD e 56 sem $\mathrm{AH} / \mathrm{SD}$, a partir do relato deles e de seus pais. Os resultados denotaram que os estudantes com AH/SD descreveram a si mesmos como mais desatentos, além de ser relatada baixa funcionalidade social. Os meninos com AH/SD relataram ter mais sintomas depressivos do que as meninas com AH/SD. Nesse estudo foi possível concluir que os estudantes com AH/SD têm indicativos de risco em relação à saúde mental.

Observa-se que há controvérsia na literatura a respeito das características sociais e emocionais de pessoas com AH/SD. Alguns estudos apontam que pessoas com AH/SD têm apresentado problemas e/ou déficits em habilidades sociais, enquanto outros não associam essa lacuna de repertório a AH/SD. A utilização de três grupos de informantes para coletar informações sobre habilidades sociais de crianças com AH/SD é pouco abordada nos artigos, o que constitui uma proposta de estudo diferenciada. A descrição das habilidades sociais e de problemas de comportamento de crianças identificadas com AH/SD, obtidas a partir de uma avaliação que envolve diferentes instrumentos e informantes de mais de um contexto, pode resultar em um conjunto expressivo de informações que ajude a ampliar o conhecimento do fenômeno.

O objetivo deste estudo foi: a) descrever e comparar o repertório de habilidades sociais de crianças com AH/SD sob o ponto de vista da criança e de seus respectivos pais/responsáveis e professoras; $b$ ) descrever e comparar as habilidades sociais e os problemas de comportamento de crianças com AH/SD sob o ponto de vista de seus pais/responsáveis e professoras, considerando o sexo e, comparar a importância das habilidades sociais para os dois grupos; e c) descrever a competência acadêmica das crianças segundo as professoras.

\section{Métodos}

Esta pesquisa foi aprovada pelo Comitê de Ética em Pesquisa (CEP) sob o no 1.184.996, em agosto de 2015. Foram cumpridos os preceitos éticos, de acordo 
com a Resolução no 510 da Comissão Nacional de Ética em Pesquisa (Conep, 2016), como a assinatura do Termo de Consentimento Livre e Esclarecido pelos pais/responsáveis e professoras, e do Termo de Assentimento Livre e Esclarecido pelas crianças.

\section{Participantes}

Participaram dez crianças com AH/SD, estudantes de uma escola estadual de ensino fundamental dos anos iniciais de uma cidade de médio porte do interior paulista. Elas foram identificadas no estudo de Mendonça (2015). Eram seis meninos e quatro meninas com idade entre 6 e 10 anos (média $=8,60$; $\mathrm{DP}=1,43$ ). Dentre elas, duas cursavam o primeiro ano; três, o terceiro; uma, o quarto; e quatro, o quinto ano.

Participaram também os pais/responsáveis: sete eram mães, uma avó e dois pais, com idade entre $30 \mathrm{e}$ 56 anos (média = 38,50; DP =9,22). Com relação ao nível acadêmico, um tinha ensino fundamental completo; cinco, o ensino médio completo; dois, o ensino superior incompleto; e dois, o ensino superior completo.

Dentre as oito professoras participantes, a idade variou entre 28 e 63 anos (média $=43,25 ; \mathrm{DP}=11,36$ ) e o tempo de serviço como professora era de 6 a 21 anos. Concernente à formação, variou do magistério ao curso de pedagogia e educação artística com pós-graduação. Todas as professoras trabalhavam em classe comum no ensino regular.

O critério de inclusão foi ter sido identificado com AH/SD no estudo de Mendonça (2015), ser aluno matriculado na escola onde foi realizada a pesquisa e os pais e professores terem disponibilidade para participar. O critério de exclusão foi não contemplar qualquer um dos três critérios de inclusão. Como a escola contempla apenas o Fundamental I, os alunos identificados no estudo de Mendonça (2015) que estavam no Fundamental II no momento da pesquisa e, consequentemente, em outra escola, não participaram.

\section{Instrumentos}

a) Sistema de Avaliação de Habilidades Sociais (Social Skills Rating System ou SSRS): é uma escala elaborada por Gresham e Elliott (1990), adaptada e validada para a amostra brasileira, que avalia habilidades sociais, problemas de comportamento e competência acadêmica (Del Prette, Del Prette, Silva, \& Bandeira, 2016). O SSRS-BR é apresentado em três versões. A versão para professores é composta por quatro fatores: responsabilidade, autocontrole, assertividade/ desenvoltura social e cooperação/afetividade; a versão para os pais é composta por cinco fatores: responsabilidade, autocontrole, afetividade/cooperação, desenvoltura social e civilidade; a versão da criança é subdivida em quatro fatores: empatia/afetividade, responsabilidade, autocontrole/civilidade e assertividade.

Os problemas de comportamento são avaliados pelos pais e professores. A versão dos pais possui dois fatores: problemas externalizantes e internalizantes; e a dos professores, problemas externalizantes, internalizantes e hiperatividade. Para cada uma das afirmações, o respondente pode assinalar: $0=$ Nunca; 1 = Algumas vezes; ou 2 = Muito frequente. Avalia-se o grau de habilidades sociais calculando-se a soma dos escores obtidos em cada item das subescalas, que pode variar entre zero e dois, transformando-os em percentil, conforme o sexo do estudante. A versão para professores e pais avalia também se as habilidades sociais são importantes e, em uma escala Likert, quão importantes são. A pontuação para cada item varia entre $0=$ Não importante; $1=$ Importante; e 2 = Indispensável.

As habilidades sociais e os problemas de comportamentos das crianças avaliadas segundo seus pais e professores são calculados separadamente, transformando os escores obtidos em percentil, conforme o sexo do estudante. Quanto mais alto o percentil na escala de habilidades sociais mais elaborado é o repertório e, quanto mais baixo o percentil na escala de problemas de comportamento, menor é sua frequência.

A validação e a adaptação para o contexto brasileiro demonstrou que o SSRS-BR possui satisfatória consistência interna, aferida pelo alfa de Cronbach nas escalas de habilidades sociais (estudante $=0,78$; pais $=0,86$; professores $=0,94$ ) e de comportamentos problemáticos (pais $=0,83$; professores $=0,91$ ).

b) Questionário de Respostas Socialmente Habilidosas para Professores (QRSH-Pr): construído por Bolsoni-Silva, Marturano e Loureiro (2009), é composto por uma lista com 24 itens de comportamentos socialmente habilidosos apresentados por crianças. Os educadores devem responder se um comportamento se aplica (escore 2), se se aplica em parte (escore 1) ou se não se aplica (escore 0). Os escores são somados, produzindo o escore total da criança avaliada, que pode variar entre $0 \mathrm{e} 48$ pontos. As propriedades psicométricas do QRSH-Prindicaram consistência interna satisfatória e validade preditiva. O coeficiente alfa, 
que corresponde à consistência de cada fator, foi alto para o fator 1: sociabilidade e expressividade emocional $(0,92)$; e para o fator 2 : iniciativa social $(0,87)$; e aceitável para o fator 3: busca de suporte $(0,73)$.

c) Questionário de Respostas Socialmente Habilidosas para Pais (QRSH-Pais): elaborado por Bolsoni-Silva, Marturano e Loureiro (2011), consiste em 18 perguntas sobre os comportamentos dos filhos. Pode ser respondido com uma de três alternativas: "não se aplica" (0 pontos - quando a criança não apresenta nunca o comportamento); "se aplica um pouco" (1 ponto - quando a criança apresenta poucas vezes determinado comportamento); e "certamente se aplica" ( 2 pontos - quando a criança apresenta frequentemente determinado comportamento). Os escores são somados, produzindo o escore total da criança avaliada, que pode variar entre 0 e 36 pontos. A consistência interna foi aferida para o escore total. $\mathrm{O}$ valor de alfa obtido foi igual a 0,82 , indicando satisfatória consistência interna do instrumento.

\section{Procedimento}

\section{Coleta dos dados}

Agendou-se uma data para a apresentação da pesquisa às professoras no horário da aula de trabalho pedagógico coletivo (ATPC) e, aos pais/responsáveis, no horário noturno, para convidá-los a participar. O estudo teve três etapas de coleta de dados. A primeira delas foi a aplicação do SSRS-BR (versão para professores) e do QRSH-Pr, em grupo, com as professoras durante o horário do ATPC. Uma professora respondeu sobre três estudantes, pois eram da mesma turma. Na segunda etapa, após a reunião de esclarecimentos e o convite aos pais/responsáveis, aplicou-se, em grupo, o SSRS-BR (versão para pais) e o QRSH-Pais. Por fim, na terceira etapa, as crianças, em horário de aula, foram convidadas a participar e responderam o SSRS-BR (versão para estudantes). Para as crianças do primeiro ano, a aplicação foi individual e a pesquisadora leu as questões e anotou as respostas. Para as demais, a aplicação foi realizada em grupo, por ano e turno escolar. A coleta com todos os participantes (crianças, professoras e pais/responsáveis) foi realizada nas dependências da escola das crianças e professoras participantes, em uma sala regularmente utilizada para o ensino de informática, livre de barulhos e outros estímulos, proporcionando privacidade a todos.

\section{Análise de dados}

As análises foram conduzidas conforme $o$ manual de cada instrumento respondido pelas crianças, pais/responsáveis e professoras. A caracterização do repertório de habilidades sociais obtido com o SSRS foi realizada considerando o percentil proposto por Del Prette et al. (2016). Neste estudo, consideraram-se os percentis de 0 a 35 como equivalentes ao repertório de habilidades sociais entre abaixo da média e médio inferior, e, para problemas de comportamento, entre muito baixo e abaixo da média. Os percentis de 36 a 100 equivaleram ao repertório de habilidades sociais entre bom e altamente elaborado, e, para problemas de comportamento, entre mediano e acima da média. Alguns fatores da escala de habilidades sociais do SSRS-BR foram agrupados para fazer comparações, pois não eram idênticos para os três respondentes; por exemplo, agrupou-se o fator autocontrole, de pais e professores, com o fator autocontrole/civilidade, de estudantes; o fator afetividade/cooperação, de pais e professores, com o fator empatia/ afetividade, de estudantes; o fator civilidade, de pais, com o fator autocontrole/civilidade de estudantes; e o fator assertividade, de estudantes, com o fator assertividade/desenvoltura social, de professores.

Para as análises com o QRHS, consideraram-se as questões iguais entre as duas versões, de pais e professores. Sendo assim, utilizaram-se todas as 18 questões da versão dos pais e 18 das 24 questões da versão dos professores.

Análises estatísticas não paramétricas foram conduzidas (teste Friedman) para comparar a frequência das habilidades sociais das crianças avaliadas pelos três respondentes. Para identificar a direção da diferença dos comportamentos habilidosos e os indicativos de problemas de comportamento com a diferença estatística no teste Friedman, foram realizadas comparações (teste Wilcoxon) com pares, dois a dois. Além disso, compararam-se as habilidades sociais que os pais/responsáveis e as professoras relataram ser importantes ou não. E, por fim, comparou-se o relato de pais/responsáveis e de professoras a respeito das habilidades sociais e dos problemas de comportamento em relação ao sexo das crianças. Considerando-se a posição do percentil, indicou-se em quais fatores as meninas e os meninos tinham habilidades e déficits sociais, segundo o seu relato, de seus pais/responsáveis e de suas 
professoras. Consideraram-se como significativas as comparações com $\mathrm{p} \leq 0,05$. Serão apresentados os dados apenas dos itens em que há diferença com significância estatística.

\section{Resultados e discussão}

A seção de resultados apresenta a caracterização de habilidades sociais e problemas de comportamento quanto a suas ocorrências (Tabela 1). A Tabela 2 explicita as comparações quanto às habilidades sociais e aos problemas de comportamento, considerando os três diferentes respondentes (professoras, pais/responsáveis e crianças) e ponderando a ocorrência de respostas. A Tabela 3 contém os resultados de meninas e meninos, além de indicativos conforme a classificação em percentil. Por sua vez, a Tabela 4 descreve os achados de acordo com o relato dos participantes quanto à importância dos comportamentos investigados pelo SSRS.
De acordo com a Tabela 1, nota-se que o repertório de habilidades sociais, no fator geral HS, foi o mais indicado (nove crianças) por pais/responsáveis com repertório entre bom e altamente elaborado, seguido pelas professoras, que apontaram, nessa condição, sete crianças. Ambos os grupos indicaram mais crianças com habilidades (percentil 36 a 100 - HS) do que com déficits (percentil 1 a 35 - HS), corroborando pesquisas cujos resultados indicam que essa população apresenta bom repertório de habilidades sociais (Bain, Choate, \& Bliss, 2006; Freitas \& Del Prette, 2013).

Os pais/responsáveis e as professoras relataram que a maioria das crianças apresentava repertório entre muito baixo e baixo no fator geral PC (percentil 0 a 35 - PC) para problemas de comportamento, indicando baixa frequência. Entretanto, entre os problemas de comportamento relatados, as professoras destacaram que mais crianças apresentavam problemas internalizantes e hiperatividade, e os pais indicaram mais problemas externalizantes.

Tabela 1

Frequência absoluta de indicação de crianças em cada fator avaliado pelo SSRS-BR considerando o percentil, segundo relato delas, de suas professoras e de seus pais/responsáveis.

\begin{tabular}{|c|c|c|c|c|c|c|}
\hline \multirow{2}{*}{$\begin{array}{l}\quad \begin{array}{c}\text { Repertório de } \\
\text { Habilidades Sociais (HS) }\end{array} \\
\text { Fatores }\end{array}$} & \multicolumn{3}{|c|}{$\begin{array}{l}\text { HS de bom a altamente elaborado } \\
\text { (Percentil 36-100) }\end{array}$} & \multicolumn{3}{|c|}{$\begin{array}{l}\text { HS de abaixo da média a } \\
\text { médio inferior } \\
\text { (Percentil 0-35) }\end{array}$} \\
\hline & Prof. & Pais & Crianças & Prof. & Pais & Crianças \\
\hline Geral HS & 7 & 9 & 4 & 3 & 1 & 6 \\
\hline Responsabilidade & 9 & 9 & 7 & 1 & 1 & 3 \\
\hline Autocontrole/Civilidade & 7 & 8 & 5 & 3 & 2 & 5 \\
\hline Assertividade/Desenvoltura social & 7 & 7 & 4 & 3 & 3 & 6 \\
\hline Afetividade/Cooperação/Empatia & 9 & 7 & 6 & 1 & 3 & 4 \\
\hline Civilidade & - & 10 & 5 & - & 0 & 5 \\
\hline $\begin{array}{l}\text { Repertório de Problemas de } \\
\text { Comportamento (PC) }\end{array}$ & \multicolumn{3}{|c|}{$\begin{array}{c}\text { PC de mediano a acima } \\
\text { da média } \\
\text { (Percentil 36-100) }\end{array}$} & \multicolumn{3}{|c|}{$\begin{array}{l}\text { PC de muito baixo } \\
\text { a baixo } \\
\text { (Percentil 0-35) }\end{array}$} \\
\hline Fatores & Prof. & Pais & Crianças & Prof. & Pais & Crianças \\
\hline Geral PC & 4 & 4 & - & 6 & 6 & - \\
\hline PC Externalizantes & 3 & 8 & - & 7 & 2 & - \\
\hline PC Internalizantes & 6 & 3 & - & 4 & 7 & - \\
\hline Hiperatividade & 6 & - & - & 4 & - & - \\
\hline
\end{tabular}

Nota. - não é avaliado pelo instrumento. 
Com relação ao autorrelato das crianças, observou-se que variam os fatores e a quantidade de crianças ao relatarem seus comportamentos habilidosos e seus déficits. Observa-se que, pela escala geral de habilidades sociais, o resultado contraria os encontrados por França-Freitas, Del Prette, Del Prette (2014) e por Loos-Sant'Ana e Trancoso (2014), que encontraram no autorrelato dos estudantes referências a boas habilidades sociais. Neste estudo, a maioria das crianças (seis) se autoavaliou com repertório entre abaixo da média e médio inferior em habilidades sociais gerais e assertividade/ desenvoltura social.

No estudo de Eren et al. (2018), ao comparar o autorrelato de estudantes com e sem AH/SD sobre aspectos sociais, os participantes com AH/SD se autoavaliaram com repertório inferior. Pessoas com AH/SD tendem a ser autocríticas (Renzulli \& Reis, 1997; Virgolim, 2016) e isso pode ter relação com as contingências estabelecidas pelos pais. Morawska e Sanders $(2008,2009)$ apontam algumas razões possíveis para que os superdotados tenham problemas comportamentais e emocionais, como as expectativas extremas e as preocupações intensas de pais e professores.

A Tabela 2 descreve as medianas, o desvio-padrão e as comparações entre as respostas do SSRS-BR e dos QRSH (pais e professores) nas escalas e fatores de habilidades sociais e de problemas de comportamento, respectivamente, respondidos pelas professoras e pais/responsáveis, assim como as das respostas do SSRS-BR respondido pelas crianças.

\section{Tabela 2}

Mediana, desvio-padrão (DP) e comparações pelos testes Friedman e Wilcoxon dos resultados do SSRS-BR, respondido pelas professoras, pais/responsáveis e crianças nas escalas de Habilidades Sociais (HS) e problemas de comportamentos (PC), e comparação pelo teste Wilcoxon do QRSH, respondido pelas professoras e pais/responsáveis.

\begin{tabular}{|c|c|c|c|c|c|c|c|}
\hline \multirow{2}{*}{ SSRS } & Prof. & $\begin{array}{l}\text { Pais/ } \\
\text { Resp. }\end{array}$ & Crianças & $\begin{array}{l}\text { Prof. } \times \text { Pais } \times \\
\text { Crianças }\end{array}$ & $\begin{array}{l}\text { Prof. } x \\
\text { Pais }\end{array}$ & $\begin{array}{c}\text { Prof. } \times \\
\text { Crianças }\end{array}$ & $\begin{array}{c}\text { Pais } \times \\
\text { Crianças }\end{array}$ \\
\hline & $\begin{array}{l}\text { mediana } \\
\text { (DP) }\end{array}$ & $\begin{array}{l}\text { mediana } \\
\text { (DP) }\end{array}$ & $\begin{array}{l}\text { mediana } \\
\text { (DP) }\end{array}$ & $\mathrm{p}$ & $\mathrm{p}$ & $\mathrm{p}$ & $\mathrm{p}$ \\
\hline Geral HS & $\begin{array}{c}34 \\
(7,6)\end{array}$ & $\begin{array}{c}32 \\
(5,92)\end{array}$ & $\begin{array}{c}24 \\
(6,34)\end{array}$ & & & & $0,011^{*}$ \\
\hline Responsabilidade & $\begin{array}{c}12 \\
(1,76)\end{array}$ & $\begin{array}{c}5 \\
(1,64)\end{array}$ & $\begin{array}{c}8,5 \\
(1,4)\end{array}$ & $0,000 *$ & $\mathbf{0 , 0 0 5 *}$ & $0,009^{*}$ & $0,011^{*}$ \\
\hline $\begin{array}{l}\text { Autocontrole/ } \\
\text { Civilidade }\end{array}$ & $\begin{array}{c}12 \\
(4,08)\end{array}$ & $\begin{array}{c}7 \\
(2,54)\end{array}$ & $\begin{array}{c}7,5 \\
(2,4)\end{array}$ & $\mathbf{0 , 0 3 8 *}$ & $\mathbf{0 , 0 2 5 *}$ & $\mathbf{0 , 0 3 2 *}$ & \\
\hline $\begin{array}{l}\text { Assertividade/ } \\
\text { Desenvoltura social }\end{array}$ & $\begin{array}{c}7 \\
(2,42)\end{array}$ & $\begin{array}{c}5 \\
(1,37)\end{array}$ & $\begin{array}{c}3 \\
(1,74)\end{array}$ & $0,046^{*}$ & & $0,021^{*}$ & $0,031^{*}$ \\
\hline $\begin{array}{l}\text { Afetividade/ } \\
\text { Cooperação/ Empatia }\end{array}$ & $\begin{array}{c}4 \\
(0,94)\end{array}$ & $\begin{array}{c}10 \\
(2,14)\end{array}$ & $\begin{array}{c}7,5 \\
(2,3)\end{array}$ & $0,001 *$ & 0,008* & $\mathbf{0 , 0 2 0 *}$ & $0,048^{*}$ \\
\hline PC Externalizantes & $\begin{array}{c}0 \\
(2,54)\end{array}$ & $\begin{array}{c}6 \\
(2,68)\end{array}$ & - & - & 0,008* & - & - \\
\hline \multicolumn{8}{|l|}{ QRSH } \\
\hline Procura sua atenção & $\begin{array}{c}1,5 \\
(0,78)\end{array}$ & $\begin{array}{c}2,0 \\
(0,00)\end{array}$ & - & - & $\mathbf{0 , 0 3 8} *$ & - & - \\
\hline
\end{tabular}

Nota. - não é avaliado pelo instrumento.

De acordo com a Tabela 2, para as habilidades sociais gerais, os pais/responsáveis identificaram que as crianças eram significativamente mais habilidosas quando seus relatos foram comparados aos dos filhos. As professoras referiram significativamente mais responsabilidade do que os pais/responsáveis e crianças; estas, por sua vez, relataram significativamente mais tais comportamentos do que seus pais/ 
responsáveis. As professoras mencionaram igualmente que as crianças emitiram mais comportamentos de autocontrole, quando comparados seus relatos aos dos pais/responsáveis; da mesma forma, elas relataram mais assertividade/desenvoltura social do que as crianças, e os pais/responsáveis mais do que as crianças. A comparação entre professoras e pais/responsáveis não apresentou diferença significativa em assertividade, diferentemente do estudo de Galloway e Porath (1997), no qual os pais relataram mais frequentemente assertividade de crianças com $\mathrm{AH} / \mathrm{SD}$ do que seus professores.

Entretanto, os pais/responsáveis alegaram mais afetividade/cooperação/empatia do que os estudantes e professoras, e as crianças mais que as professoras, contrariando novamente o resultado de Galloway e Porath (1997), no qual os professores avaliaram a cooperação dos estudantes com AH/SD mais frequentemente do que os pais. Do mesmo modo, os pais/ responsáveis descreveram mais problemas de comportamento externalizantes do que as professoras; todavia, ambos grupos relataram igualmente problemas de comportamento internalizantes e de habilidades sociais em geral, corroborando Galloway e Porath (1997), que não encontraram diferenças significativas na comparação entre as avaliações dos professores e as de pais de estudantes com AH/SD na escala geral.

Distintivamente de Galloway e Porath (1997), que encontraram diferenças significativas entre o relato de professores e o de pais em relação a cooperação e assertividade, este estudo encontrou-as em quatro fatores: responsabilidade, autocontrole, afetividade/cooperação e problemas de comportamento externalizantes. Bolsoni-Silva e Loureiro (2016) destacaram que as professoras relatam mais habilidades nos estudantes do que os pais, confirmando os dados desta pesquisa quanto aos fatores assertividade/desenvoltura social, bem como em relação aos de responsabilidade e autocontrole/civilidade estes dois últimos com diferenças estatisticamente significativas. Uma hipótese é que as mães são mais exigentes em suas avaliações, conforme identificado por Bolsoni-Silva et al. (2006). Com relação aos problemas de comportamento, Bandeira et al. (2006) descreveram que os pais observaram mais problemas do que os professores, dado também encontrado nesta pesquisa em relação aos problemas de comportamento geral e externalizantes, este último com diferença significativa.
No estudo de Eklund et al. (2015), ao comparar comportamentos internalizantes e externalizantes de crianças com e sem AH/SD e com e sem riscos emocionais e comportamentais, os autores não encontraram diferenças estatísticas entre o relato de pais e o dos professores. Porém, os autores destacaram diferenças quando os pais avaliaram um número maior de crianças com AH/SD com riscos, em comparação com os professores, bem como indicaram taxas mais altas de comportamentos internalizantes em crianças com AH/SD. Nesta pesquisa também não houve diferença entre o relato de pais e o de professores em relação aos comportamentos internalizantes. Entretanto, os pais indicaram taxas mais altas de comportamentos externalizantes.

Com relação ao QRSH, observa-se que somente a variável "procura sua atenção" apresentou diferença estatística significativa, sendo mais pontuada pelos pais/responsáveis. Na literatura que compara o relato de pais com o de professores são encontrados resultados diferentes, o que fortalece a importância de ter múltiplos informantes, como apontam Eklund et al. (2015), segundo os quais as experiências emocionais são diferentes conforme o contexto, de modo que cada informante é capaz de fazer contribuições relevantes.

Na Tabela 3 é exibida a média dos escores e o percentil das meninas e dos meninos, segundo as crianças e seus pais/responsáveis e professoras, obtidos no SSRS-BR. Observa-se que o relato das professoras apontou para a necessidade de atenção preventiva nos fatores de problemas de comportamento internalizantes e de hiperatividade para meninas e meninos, e em problemas de comportamento geral e externalizantes para os meninos, além da necessidade de intervenção em assertividade/desenvoltura social para as meninas.

A assertividade enquadra comportamentos que envolvem algum risco de reação indesejável do outro, como "questionar de forma educada as regras que considera injustas" (professores). A desenvoltura social envolve os comportamentos que expressam traquejo nas relações interpessoais, por exemplo, "apresenta-se a novas pessoas sem precisar mandar" (professores) (Del Prette et al., 2016). Existe uma diferença culturalmente aceita quanto aos comportamentos valorizados para meninos e meninas: às meninas são ensinados comportamentos de submissão, especialmente diante da coerção, de modo que aprendam, então, a se comportar de forma passiva diante de interações negativas (Leman \& Björnberg, 2010), o que pode justificar as professoras notarem e relatarem como déficit nessa área. 
Tabela 3

Resultados das médias dos escores de meninas e meninos obtidos no instrumento SSRS-BR e indicativos conforme classificação em percentil.

\begin{tabular}{|c|c|c|c|c|c|c|}
\hline \multirow{3}{*}{ Fatores } & \multicolumn{2}{|c|}{ Professoras } & \multicolumn{2}{|c|}{ Crianças } & \multicolumn{2}{|c|}{ Pais/Resp. } \\
\hline & Meninas & Meninos & Meninas & Meninos & Meninas & Meninos \\
\hline & $\begin{array}{c}\text { média } \\
\text { (percentil) }\end{array}$ & $\begin{array}{c}\text { média } \\
\text { (percentil) }\end{array}$ & $\begin{array}{c}\text { média } \\
\text { (percentil) }\end{array}$ & $\begin{array}{c}\text { média } \\
\text { (percentil) }\end{array}$ & $\begin{array}{c}\text { média } \\
\text { (percentil) }\end{array}$ & $\begin{array}{c}\text { média } \\
\text { (percentil) }\end{array}$ \\
\hline Geral HS & $32(45)$ & $32(60)$ & $27(40)$ & $25(35)^{* *}$ & $34(75)$ & $31(60)$ \\
\hline Responsabilidade & $12(100)$ & $11(65)$ & $8(45)$ & $8(50)$ & $5(70)$ & $5(80)$ \\
\hline Autocontrole/Civilidade & $12(60)$ & $10(50)$ & $7(35)^{* *}$ & $7(35)^{* *}$ & $8(85)$ & $6(70)$ \\
\hline $\begin{array}{l}\text { Assertividade/ } \\
\text { Desenvoltura social }\end{array}$ & $5(35)^{* *}$ & $8(80)$ & $4(55)$ & $4(65)$ & $5(55)$ & $5(55)$ \\
\hline $\begin{array}{l}\text { Afetividade/Cooperação/ } \\
\text { Empatia }\end{array}$ & $4(50)$ & $4(55)$ & $8(50)$ & $6(25)^{* *}$ & $9(60)$ & $9(65)$ \\
\hline Civilidade & - & - & - & - & $8(100)$ & $7(50)$ \\
\hline Geral PC & $2(30)$ & $7(50)^{*}$ & - & - & $8(30)$ & $9(40)^{*}$ \\
\hline PC Externalizantes & $0(1)$ & $2(40)^{*}$ & - & - & $5(30)$ & $6,5(45)^{*}$ \\
\hline PC Internalizantes & $2(55)^{*}$ & $2(50)^{*}$ & - & - & $2(25)$ & $2,5(35)$ \\
\hline Hiperatividade & $1(45)^{*}$ & $3(55)^{*}$ & - & - & - & - \\
\hline Competência acadêmica & $42(70)$ & $42(75)$ & - & - & - & - \\
\hline
\end{tabular}

Nota. - não é avaliado pelo instrumento; * Indicativo de atenção preventiva (percentil 36 a 65 nos fatores de PC); ** Indicativo de necessidade de treinamento de HS (percentil 1 a 35 nos fatores de HS).

Nota-se que, em problemas de comportamento, os meninos apresentaram necessidade de atenção preventiva mais do que as meninas, segundo as professoras e pais, o que vai de encontro à literatura que compara gênero (Bolsoni-Silva \& Loureiro, 2016; Eklund et al., 2015).

Ao observar a autoavaliação das crianças nesta pesquisa, as meninas se identificaram com melhor repertório em habilidades sociais gerais do que os meninos; entretanto, nos comportamentos mais direcionados à relação com pares, como civilidade, meninas e meninos relataram repertório abaixo da média, com necessidade de intervenção, o que não foi encontrado em assertividade/desenvoltura social. Ao examinar todos os comportamentos, os meninos relataram baixo repertório em três áreas (habilidades sociais gerais, civilidade/autocontrole e cooperação/empatia) e as meninas em apenas uma (civilidade/autocontrole). As diferenças de gênero são descritas também pelas próprias crianças, confirmando os achados de Eren et al. (2018) com meninos e meninas com e sem AH/SD. Os meninos se identificaram por meio do autorrelato com mais problemas entre os pares do que as meninas; estas, por sua vez, também de ambos os grupos, se identificaram com mais comportamentos sociais do que os meninos.

Com relação à competência acadêmica, as professoras avaliaram-na como acima da média para ambos os grupos, meninas e meninos, o que era esperado, pois as crianças foram identificadas com AH/SD por Mendonça (2015) por meio de uma avaliação multimodal, inclusive de avaliações acadêmicas (Mendonça, Rodrigues, \& Capellini, 2017, 2018).

A Tabela 4 apresenta as medianas, desvio-padrão e comparações do instrumento Sistema de Avaliação de Habilidades Sociais (SSRS-BR) na escala e nos fatores de frequência e de importância das habilidades sociais de meninos, respondido por suas professoras e pais/responsáveis. As mesmas comparações foram realizadas para as meninas, porém não houve diferença com significância estatística. 


\section{Tabela 4}

Mediana, desvio-padrão (DP) e comparações pelo teste Wilcoxon no instrumento SSRS-BR, respondido pelas professoras e pais/responsáveis de meninos nas escalas de frequência e importância de habilidades sociais (HS) e de frequência de problemas de comportamento (PC).

\begin{tabular}{|c|c|c|c|c|c|c|}
\hline \multirow{4}{*}{ Fatores } & \multicolumn{5}{|c|}{ Meninos } & \\
\hline & \multicolumn{2}{|c|}{ Frequência } & & \multicolumn{2}{|c|}{ Importância } & \\
\hline & Pais & Prof. & & Pais & Prof. & \\
\hline & mediana (DP) & & $\mathrm{p}$ & mediana (DP) & & $\mathrm{p}$ \\
\hline Responsabilidade & $4,5(2,05)$ & $11,5(2,14)$ & 0,028* & $5,5(0,74)$ & $7(3,00)$ & \\
\hline Afetividade/Cooperação & $9(1,75)$ & $4,5(1,10)$ & $0,042^{*}$ & $10,5(1,34)$ & $3(1,46)$ & $0,042^{*}$ \\
\hline PC Externalizante & $6(2,69)$ & $0,5(2,98)$ & $0,046^{*}$ & - & - & - \\
\hline
\end{tabular}

Nota. - não é avaliado pelo instrumento.

As professoras opinaram que os meninos apresentavam mais comportamentos de responsabilidade, em comparação com os relatos de pais/ responsáveis. Os pais/responsáveis relataram que os meninos apresentavam mais afetividade/cooperação e consideraram esses comportamentos mais importantes do que as professoras. Os pais/responsáveis, do mesmo modo, apontaram que os meninos apresentavam mais problemas de comportamento externalizantes, em comparação com respostas das professoras, conforme destacaram Bandeira et al. (2006) ao afirmar que pais notam mais problemas de comportamento do que professores.

\section{Considerações finais}

Este estudo possibilitou avaliar as habilidades sociais e os problemas de comportamento de crianças com AH/SD, concluindo que é necessária atenção preventiva e intervenção nos fatores que apresentam déficits sociais, de acordo com o relato das crianças, seus respectivos pais/responsáveis e professoras, ainda que as crianças apresentem habilidades sociais. Desenvolver essas habilidades é importante, pois, de acordo com França-Freitas, Del Prette e Del Prette (2017), quanto maior o repertório de habilidades sociais, maior é a qualidade de vida, com relações interpessoais mais gratificantes, maior realização pessoal e sucesso profissional, além de melhor saúde física e mental.

Destacam-se alguns pontos fortes desta pesquisa: a) além do autorrelato das crianças, a participação de múltiplos informantes que convivem com elas em diferentes contextos: as professoras na escola e seus pais/responsáveis em casa e em outros ambientes, como parques, shopping, igreja etc.; b) há poucas pesquisas na área de AH/SD com crianças, sobretudo sobre questões emocionais e sociais, tornando este estudo uma contribuição para a literatura da área; c) a maioria dos estudos que avaliam as habilidades sociais examina apenas a frequência. Além da frequência das habilidades, este estudo avaliou a importância delas para pais/responsáveis e professoras, e a frequência de problemas de comportamento; e d) o fato de dois pais terem participado do estudo também é relevante, pois pesquisas que avaliam os filhos são na maioria conduzidas com mães.

Entre as limitações deste estudo, uma foi não avaliar o desempenho acadêmico e os problemas de comportamento das crianças segundo seu relato, o que poderia ter sido feito utilizando-se outros instrumentos. Outra limitação foi o número reduzido de participantes, o que restringe a possibilidade de generalizações dos resultados. Além disso, o estudo não teve um grupo-controle para comparação dos resultados. Para pesquisas futuras, recomenda-se coletar dados com um número maior de participantes, bem como acrescentar um grupo-controle. Do mesmo modo, sugere-se que se investigue o desempenho acadêmico, os problemas de comportamento e a importância das habilidades sociais a partir do relato da criança. Tais dados possibilitam que sejam programadas intervenções preventivas, conforme a indicação dos pais/responsáveis e das professoras, e condizente com as necessidades apontadas pelas próprias crianças. 


\section{Referências}

Alencar, E. M. L. S. (2007). Características sócio-emocionais do superdotado: questões atuais. Psicologia em Estudo, 12(2), 371-378. https://doi.org/10.1590/S1413-73722007000200018

Almeida, I. S., Rocha, A., \& Fonseca, H. (2016). Programa Parentalidade Positiva: Programa de intervenção parental de crianças e jovens sobredotados. Sobredotação, 15(1), 113-131.

Antipoff, C. A., \& Campos R. H. F. (2010). Superdotação e seus mitos. Psicologia Escolar e Educacional, 14(2), 301-309. https://doi.org/10.1590/S1413-85572010000200012

Bain, S. K., Choate, S. M., \& Bliss, S. L. (2006). Perceptions of developmental, social, and emotional issues in giftedness: Are they realistic? Roeper Review, 29(1), 41-48. https:// doi.org/10.1080/02783190609554383

Bandeira, M., Silva R. S., Cordeiro F. L., Del Prette, Z. P., \& Del Prette, A. (2006). Habilidades sociais e variáveis sociodemográficas em estudantes do ensino fundamental. Psicologia em Estudo, 11(3), 541-549. https://doi.org/ $10.1590 /$ S1413-73722006000300010

Barreto, S. O., Freitas, L. C., \& Del Prette, Z. A. P. (2011). Habilidades sociais na comorbidade entre dificuldades de aprendizagem e problemas de comportamento: Uma avaliação multimodal. Psico, 42(4), 503-510.

Bolsoni-Silva, A. T., \& Loureiro, S. R. (2016). Simultaneous assessement of social skills and behavior problems: education and gender. Estudos de Psicologia, 33(3), 453-464. https://doi.org/10.1590/1982-02752016000300009

Bolsoni-Silva, A. T., Marturano, E. M., \& Loureiro, S. R. (2009). Construction and validation of the Brazilian Questionário de Respostas Socialmente Habilidosas segundo relato de professores (QRSH-PR). The Spanish Journal of Psychology, 12(1), 349-359. https://doi.org/10.1017/S1138741600001748

Bolsoni-Silva, A. T., Marturano, E. M., \& Loureiro, S. R. (2011). Estudos de confiabilidade e validade do questionário de respostas socialmente habilidosas versão para pais - QRSH-Pais. Psicologia: Reflexão e Crítica, 24(2), 227-235. https://doi.org/10.1590/S0102-79722011000200003

Bolsoni-Silva, A. T., Marturano, E. M., Pereira, V. A., \& Manfrinato, J. W. S. (2006). Habilidades sociais e problemas de comportamento de pré-escolares: Comparando avaliações de mães e de professoras. Psicologia: Reflexão $e$ Crítica, 19(3), 460-469. https:// dx.doi.org/10.1590/S0102-79722006000300015

Chagas, J. F., \& Fleith, D. S. (2010). Habilidades, características pessoais, interesses e estilos de aprendizagem de adolescentes talentosos. Psico-USF, 15(1), 93-102. https://doi.org/10.1590/S1413-82712010000100010

Conselho Nacional de Saúde. (2016). Resolução no 510, de 7 de abril de 2016. Ministério da Saúde. http:// conselho. saude.gov.br/resolucoes/2016/Reso510.pdf

Del Prette, A., \& Del Prette, Z. A. P. (2008). Psicologia das relações interpessoais: Vivências para o trabalho em grupo. Vozes.

Del Prette, A., \& Del Prette, Z. A. P. (2017). Competência social e habilidades sociais: Manual teórico-prático. Vozes.

Del Prette, Z. A. P., \& Del Prette, A. (2009). Avaliação de habilidades sociais: Bases conceituais, instrumentos e procedimentos. In Z. A. P. Del Prette, \& A. Del Prette (Orgs.), Psicologia das habilidades sociais: Diversidade teórica e suas implicações (pp. 189-231). Vozes.

Del Prette, Z. A. P., \& Del Prette, A. (2013). Psicologia das habilidades sociais na infância: Teoria e prática. Vozes.

Del Prette, Z. A. P., Del Prette, A., Silva, L. C., \& Bandeira. M. (2016). Inventário de habilidades sociais, problemas de comportamento e competência acadêmica para crianças - SSRS: Manual de aplicação, apuração e interpretação. Casa do Psicólogo.

Eklund, K., Tanner N., Stoll, K., \& Anway, L. (2015). Identifying emotional and behavioral risk among gifted and nongifted children: A multi-gate, multi-informant approach. School Psychology Quarterly, 30(2), 197-211. http://doi.org/10.1037/spq0000080

Eren, F., Çete, A. O., Avcil, S., \& Baykara, B. (2018). Emotional and behavioral characteristics of gifted children and their families. Noro Psikiyatri Arsivi, 55(2), 105- https://doi.org/112. 10.5152/npa.2017.12731

França-Freitas, M. L. P., Del Prette, A., \& Del Prette, Z. A. P. (2014). Social skills of gifted and talented children. Estudos de Psicologia, 19(4), 288-295. https://doi.org/10.1590/S1413-294X2014000400006

França-Freitas, M. L. P., Del Prette, A., \& Del Prette, Z. A. P. (2017). Habilidades sociais e bem-estar subjetivo de crianças dotadas e talentosas. Psico-USF, 22(1), 1-12. https://doi.org/10.1590/1413-82712017220101

Freitas, L. C., \& Del Prette, Z. A. P. (2013). Habilidades sociais de crianças com diferentes necessidades educacionais especiais: Avaliação e implicações para intervenção. Avances en Psicología Latinoamericana, 31(2), 344-362. 
Galloway, B., \& Porath, M. (1997). Parent and teacher views of gifted children's social abilities. Roeper Review, 20(2), 118-121. https:// doi.org/10.1080/02783199709553872

Gauck, L., \& Trommsdorff, G. (2009). Probleme hochbegabter Kinder aus Sicht von Kindern, Eltern und Lehrern. Psychologie in Erziehung und Unterricht, 56(1), 27-37.

Gokaydin, B., \& Ozcan D. (2018). Social and emotional problems faced by gifted individuals. IIOAB Journal, 9(3), 30-43.

Gresham, F. M. (2009). Análise do comportamento aplicado às habilidades sociais. In Z. A. P. Del Prette \& A. Del Prette (Orgs.), Psicologia das habilidades sociais: Diversidade teórica e suas implicações (pp. 17-56). Vozes.

Gresham, F. M., \& Elliott, S. N. (1990). Social skills rating system: Manual. American Guidance Service.

Leman, P. J., \& Björnberg, M. (2010). Conversation, development, and gender: A study of changes in children's concepts of punishment. Child Development, 81(3), 958-971. https://doi.org/10.1111/j.1467-8624.2010.01445.x

Loos-Sant'Ana, H., \& Trancoso, B. S. (2014). Socio-emotional development of Brazilian gifted children: Selfbeliefs, social skills, and academic performance. Journal of Latino/Latin American Studies, 6(1), 54-65. https://doi.org/ 10.18085/llas.6.1.g438862334102t30

McFall, R. M. (1982). A review and reformulation of the concept of social skills. Behavioral Assessment, 4(1), 1-33. http://doi.org/10.1007/BF01321377

Mendonça, L. D. (2015). Identificação de alunos com altas habilidades ou superdotação a partir de uma avaliação multimodal [Dissertação de mestrado, Universidade Estadual Júlio de Mesquita Filho]. Repositório Institucional Unesp. https://repositorio.unesp.br/handle/11449/124021

Mendonça, L. D., Rodrigues, O. M. P. R., \& Capellini, V. L. M. F. (2017). Identificação inicial de alunos com altas habilidades ou superdotação: Avaliação intelectual, de desempenho escolar e indicação pelos professores. Revista de Educação Especial e Reabilitação, 30(57), 203-218. http:/ /dx.doi.org/10.5902/1984686X24120

Mendonça, L. D., Rodrigues, O. M. P. R., \& Capellini, V. L. M. F. (2018). WISC-III: Instrumento para Confirmação de Altas Habilidades/Superdotação. Psicologia: Ciência e Profissão, 38(1), 52-60. https://doi.org/ $10.1590 / 1982-3703001062017$

Morawska, A., \& Sanders, M. R. (2008). Parenting gifted and talented children: What are the key child behaviour and parenting issues? Australian and New Zealand Journal of Psychiatry, 42(9), 819-827. https://doi.org/ $10.1080 / 00048670802277271$

Morawska, A., \& Sanders, M. R. (2009). An evaluation of a behavioral parenting intervention for parents of gifted children. Behaviour Research and Therapy, 47(6), 463-479. https://doi.org/10.1016/j.brat.2009.02.008

Ogurlu, U., Yalin, H. S., \& Birben, F. Y. (2018). The relationship between psychological symptoms, creativity, and loneliness in gifted children. Journal for the Education of the Gifted, 41(2), 193-210. https://doi.org/ $10.1177 / 0162353218763968$

Oliveira, A. P., Capellini, V. L. M. F., \& Rodrigues, O. M. P. R. (2020). Altas habilidades/superdotação: Intervenção em habilidades sociais com estudantes, pais/responsáveis e professoras. Revista Brasileira de Educação Especial, 26(1), 125-142. https://doi.org/10.1590/s1413-65382620000100008

Patterson, G., Reid, J., \& Dishion, T. (2002). Antisocial boys: Comportamento antissocial. ESETec.

Peterson, S. J. (2009). Myth 17: Gifted and talented individuals do not have unique social and emotional needs. Gifted Child Quarterly, 53(4), 280-282. https://doi.org/10.1177/0016986209346946

Prieto, M. D., Ferrándiz, C., Ferrando, M., Sánchez, C., \& Bermejo, R. (2016). Inteligencia emocional y alta habilidad. Sobredotação, 15(1), 35-56.

Renzulli, J. S. (1998). The three-ring conception of giftedness. In S. M. Baum, S. M. Reis, \& L. R. Maxfield (Eds.), Nurturing the gifts and talents of primary grade students: Mansfield Center (pp. 50-72). Creative Learning.

Renzulli, J. S. (2004). O que é esta coisa chamada superdotação, e como a desenvolvemos? Uma retrospectiva de 25 anos. Educação, 1(52), 75-131.

Renzulli, J. S. (2014). A concepção de superdotação no modelo dos três anéis: Um modelo de desenvolvimento para a promoção da produtividade criativa. In A. M. R. Virgolim, \& E. C. Konkiewitz (Orgs.), Altas habilidades/ superdotação, inteligência e criatividade (pp. 219-264). Papirus.

Renzulli, J. S. (2018). Reexaminando o papel da educação para superdotados e o desenvolvimento de talentos para o século XXI: Uma abordagem teórica em quatro partes. In A. Virgolim (Org.), Altas habilidades/superdotação: Processos criativos, afetivos e desenvolvimento de potenciais (pp. 19-42). Juruá. 
Renzulli, J. S., \& Reis, S. (1997). The schoolwide enrichment model: A how-to guide for educational excellence. Creative Learning.

Richards, J., Encel, J., \& Shute, R. (2003). The emotional and behavioral adjustment of intellectually gifted adolescents:A multi-dimensional, multi-informant approach. High Ability Studies, 14(2), 153-164.https://doi.org/ $10.1080 / 1359813032000163889$

Secretaria de Educação Especial. (2008). Política nacional de educação especial na perspectiva da educação inclusiva. Ministério da Educação. http://portal.mec.gov.br/arquivos/pdf/politicaeducespecial.pdf

Versteynen, L. (2001). Issues in the social and emotional adjustment of gifted children: What does the literature say? The New Zealand Journal of Gifted Education, 13(1), 1-8.

Virgolim, A. M. R. (2016). Questões afetivas e emocionais das pessoas com altas habilidades/superdotação [Artigo apresentado]. $7^{\circ}$ Encontro Nacional do Conselho Brasileiro para Superdotação: Altas Habilidades/SuperdotaçãoDireitos, Práticas e Inovações, Bonito, MS, Brasil.

\section{Ana Paula de Oliveira}

Doutoranda e mestre em psicologia do desenvolvimento e aprendizagem pela Faculdade de Ciências da Universidade Estadual Paulista (Unesp), Bauru - SP. Brasil.

E-mail: ana_paula_apo@hotmail.com

(1) https://orcid.org/0000-0001-5044-6551

\section{Vera Lucia Messias Fialho Capellini}

Professora adjunta do Departamento de Educação, do Programa de Pós-Graduação em Psicologia do Desenvolvimento e Aprendizagem e do Programa em Docência para a Educação da Faculdade de Ciências da Universidade Estadual Paulista (Unesp), Bauru - SP. Brasil.

E-mail: vera.capellini@unesp.br

(1) https://orcid.org/0000-0002-9184-8319

\section{Olga Maria Piazentin Rolim Rodrigues}

Professora adjunta do Departamento de Psicologia e do Programa de Pós-Graduação em Psicologia do Desenvolvimento e Aprendizagem da Faculdade de Ciências da Universidade Estadual Paulista (Unesp), Bauru SP. Brasil.

E-mail: olgarolim@fc.unesp.br

(1) https://orcid.org/0000-0002-5332-256X

\section{Alessandra Turini Bolsoni-Silva}

Professora adjunta do Departamento de Psicologia e do Programa de Pós-Graduação em Psicologia do Desenvolvimento e Aprendizagem da Faculdade de Ciências da Universidade Estadual Paulista (Unesp), Bauru SP. Brasil.

E-mail: bolsoni.silva@unesp.br

D https://orcid.org/0000-0001-8091-9583

Este artigo apresenta parte dos resultados da dissertação de mestrado da autora Ana Paula de Oliveira, com a colaboração das demais. O estudo teve o apoio financeiro da Fundação de Amparo à Pesquisa do Estado de São Paulo (Fapesp). Processo no 2014/10826-6.

Endereço para envio de correspondência:

Vera Lucia Messias Fialho Capellini. Av. Eng. Luís Edmundo Carrijo Coube, 2085, Núcleo Res. Pres. Geisel. CEP:17033-360. Bauru - SP, Brasil. 
Recebido 06/02/2019

Aceito 23/07/2020

Received 02/06/2019

Approved 07/23/2020

Recibido 06/02/2019

Aceptado 23/07/2020

Como citar: Oliveira, A. P., Capellini, V. L. M. F., Rodrigues, O. M. P. R., \& Bolsoni-Silva, A. T. (2021). Habilidades sociais e problemas de comportamento de crianças com altas habilidades/superdotação. Psicologia: Ciênciae Profissão, 41, 1-17. https:// doi.org/10.1590/1982-3703003219590

How to cite: Oliveira, A. P., Capellini, V. L. M. F., Rodrigues, O. M. P. R., \& Bolsoni-Silva, A. T. (2021). Social Skills and behavioral problems of high-ability/gifted children. Psicologia: Ciência e Profissão, 41, 1-17. https://doi.org/ $10.1590 / 1982-3703003219590$

Cómo citar: Oliveira, A. P., Capellini, V. L. M. F., Rodrigues, O. M. P. R., \& Bolsoni-Silva, A. T. (2021). Habilidades Sociales y problemas conductuales de niños con altas habilidades/superdotados. Psicologia: Ciência e Profissão, 41, 1-17. https:// doi.org/10.1590/1982-3703003219590 